\title{
Criteria for fluxon generation in long Josephson junctions by current pulses
}

\author{
Sakai, S.; Samuelsen, Mogens Rugholm
}

Published in:

Applied Physics Letters

Link to article, DOI:

$10.1063 / 1.97933$

Publication date:

1987

Document Version

Publisher's PDF, also known as Version of record

Link back to DTU Orbit

Citation (APA):

Sakai, S., \& Samuelsen, M. R. (1987). Criteria for fluxon generation in long Josephson junctions by current pulses. Applied Physics Letters, 50(16), 1107-1109. https://doi.org/10.1063/1.97933

\section{General rights}

Copyright and moral rights for the publications made accessible in the public portal are retained by the authors and/or other copyright owners and it is a condition of accessing publications that users recognise and abide by the legal requirements associated with these rights.

- Users may download and print one copy of any publication from the public portal for the purpose of private study or research.

- You may not further distribute the material or use it for any profit-making activity or commercial gain

- You may freely distribute the URL identifying the publication in the public portal

If you believe that this document breaches copyright please contact us providing details, and we will remove access to the work immediately and investigate your claim. 


\title{
Criteria for fluxon generation in long Josephson junctions by current pulses
}

\author{
S. Sakai" and M. F. Samuelsen \\ Physics Laboratory I, The Technical University of Denmark, DK-2800 Lyngby, Denmark
}

(Received 19 December 1986; accepted for publication 27 January 1987)

\begin{abstract}
In recent measurements in the time domain on the fluxon shape in long Josephson junctions the fluxons were generated by a current pulse injected into one end. We present here a perturbation treatment of the fluxon gereration which we compare witr numerical experiments. The agreement turns out to be excellent.
\end{abstract}

There has been considerable interest in soliton propagation in various physical systems. One system where soliton propagation is most accessible for experimental measurements is the long Josephson junction as is well described in recent books. "In the Josephson junction the physical manifestation of a soliton is a fluxon, i.e., a moving quantum of magnetic flux $h / 2 e$ in the junction. A rather indirect, but simple way of measuring moving fuxons in the long Josephson junction is to measure the so-called zero-field steps (ZFS) in the dc current-voltage (IV) curve. The first ZFS is explained as a resonant motion of a single furxon along the junction. Recently measurement in the time domain on the fuxon shape in long Josephson junctions has been made in the experiment of fluxon devices, ${ }^{2}$ in which a fluxon is generated by a current pulse injected into one end of the transmission line, and the shape of the soliton is measured using a Josephson sampler with a time resolution of about $5 \mathrm{ps.}$

The plirpose of the letter is to investigate theoretically the fuxon generation process by a perturbation treatment and to compare the obtained results with results of numerical experiments performed on the same process.

First the method used in the experiments of Ref. 2 is reviewed. A dc current $I_{\mathrm{dc}}$ is applied to one end of a long Josephson junction $\left(I_{\mathrm{dc}}<I_{e}^{0}\right)$, which makes an initial state where a fluxon can be easily generated by small external sources. Here $I_{c}^{0}$ is the thresinold current to keep the junction in the zero voltage state, and is given by $I_{e}^{0}=2 \lambda_{J} w h_{0} . \lambda_{J}$ is the Josephson penetration depth, $w$ is the width of the long junction $\left(w<\lambda_{j}\right)$, and $I_{0}$ is the critical current density of the junction. If a pulse current in such state is applied, then a fluxon can be generated. Using this method fiuxon devices such as an oscillator and a signal divider were operated successfully. ${ }^{2}$

A semi-infinite $(0 \leqslant x<\infty)$ Josephson transmission line with current injection source to the end gives a good model for such experiments. The dynamic of the macroscopic quantum phase difference $\Phi(x, i)$ across the oxide layer is governed by a perturbed sine-Gordon equation (in normalized form) ${ }^{3}$

$$
\Phi_{x x}-\Phi_{t t}-\sin \Phi=\alpha \Phi_{t}-\beta \Phi_{x x t} .
$$

The spatial variable is measured in units of $\lambda_{J}=(\mathrm{h} /$ $\left.2 e L I_{0}\right)^{1 / 2}$ and the time in units of the reciprocal plasma frequency $\omega_{0}{ }^{1}=\left(2 e I_{0} / h C\right)^{-1 / 2}$, where $L$ is the sheet induc-

\footnotetext{
a) Permanent address: Electrotechnical Laboratory, 1-1-4 Umezono, Sakura-mura, Nihari-gun, Ibaraki 305, Japan.
}

tance of the Josephson transmission line and $C$ is the shunt capacitance per unit area. The shunt loss parameter $\alpha$ is given by $\alpha=G\left(\hbar / 2 e I_{0} C\right)^{1 / 2}$, where $G$ is the shunt conductance per unit area. The surface loss parameter $\beta$ is given by $\beta=\omega_{0} L / R_{J}$, where $R_{J}$ is the surface resistance. The injected current $I(t)$ determines the boundary condition on $\Phi$ through ${ }^{4}$

$$
\Phi_{x}(0, t)+\beta \Phi_{x t}(0, t)=-\kappa(t)
$$

where the normalized current $\kappa(t)=2 I(t) / I_{\mathrm{e}}{ }^{0}$ and in this model $\kappa(t)$ has a de part and a pulse part, i.e., $\kappa(t)=\kappa_{\mathrm{dc}}$ $+k_{P}(t)$.

The solution of the initial state before applying the pulse is given exactly by ${ }^{5}$

$$
\Phi=4 \tan ^{-1} \exp \left[-\left(x-X_{0}\right)\right] \text {, }
$$

where $X_{0}(<0)$ is related to the dc bias $\kappa_{\mathrm{dc}}$ as

$$
\kappa_{\mathrm{dc}}=2 \operatorname{sech}\left(X_{0}\right) \text {. }
$$

In the perturbation treatment we assume that $\Phi$ is represented by the following trial function at any time:

$$
\Phi(x, t)=4 \tan ^{-1} \exp \{-\gamma(u)[x-X(t)]\}
$$

and

$$
u=\frac{d X}{d t}
$$

where $X(t)$ is the position of the center of the soliton, $u$ is the velocity of the soliton, and $\gamma(u)$ is the Lorentz factor

$$
\gamma(u)=\left(1-u^{2}\right)^{1 / 2} \text {. }
$$

From Eqs. (3) and (5), the initial state is a tail of a resting fluxon centered outside the transmission line at $X(-\infty)$ $=X_{0}<0$.

According to our model this fluxon tries to move into the junction during the pulse application, and generation or nongeneration of a fuxon is determined by the following energetic consideration. Although the modei is rather crude, it gives quantitatively good result for the criteria of the fluxon generation as will be shown later.

First we define the energy $H$ in the transmission line $(0 \leqslant x<\infty)$ as

$$
H=\int_{0}^{\infty}\left(\frac{1}{2} \Phi_{x}^{2}+\frac{1}{2} \Phi_{t}^{2}+(1-\cos \Phi)\right) d x .
$$

Differentiating Eq. (8) with respect to time and using Eq. (1) and the boundary condition Eq. (2) we get without any approximation 


$$
\begin{gathered}
\frac{d H Y}{d t}=\left.\Phi_{t}\right|_{x=0} \kappa(t)-\alpha \int_{t}^{\infty} \Phi_{t}^{2} d x \\
-\beta \int_{0}^{\infty} \Phi_{x t}^{2} d x .
\end{gathered}
$$

We now put the trial function Eq. (5) into Eq. (8) to calculate the energy. The result is

$$
H=4 \gamma[1+\tanh (\gamma X)] .
$$

In this calculation, we assume that $u$ changes slowly to satisfy the following condition:

$$
\gamma^{2}\left|\frac{d u}{d t}\right| \ll 1
$$

which makes the calculation of $\Phi_{t}$ in Eq. (8) simple. Putting the trial function Eq. (5) into the right-hand side of Eq. (9) and using Eq. (11) we obtain

$$
\begin{aligned}
\frac{d H}{d t}= & 2 \gamma u \operatorname{sech}(\gamma X) \kappa(t)-4 \alpha \gamma u^{2}[1+\tanh (\gamma X)] \\
& -\frac{4}{3} B \gamma^{3} u^{2}\left[1+\tanh ^{3}(\gamma X)\right] .
\end{aligned}
$$

Finally introducing $H$ from Eq. (10) into the left-hand side of Eq. (12)-again using the condition Eq. (11) -we get an equation of motion for $u$

$$
\frac{d u}{d t}=f_{1}+\alpha f_{2}+\beta f_{3}+\kappa(t) f_{4}
$$

where

$$
\begin{aligned}
& f_{1}=-\operatorname{sech}^{2}(\gamma X) /\{\gamma[1+\tanh (\gamma X)]\} \\
& f_{2}=-u / \gamma^{2} \\
& f_{3}=-\frac{u}{3} \frac{1+\tanh ^{3}(\gamma X)}{1+\tanh (\gamma X)}
\end{aligned}
$$

and

$$
f_{4}=\frac{\operatorname{sech}(\gamma X)}{2 \gamma^{2}[1+\tanh (\gamma X)]} .
$$

Equations (6) and (13) form two first-order coupled differential equations which together with the initial condition

$$
u(-\infty)=0
$$

and [see Eq. (4)]

$$
X(-\infty)=X_{0}
$$

completely define the problem.

We now solve Eqs. (6), (13), and (15) and use as criteria for a fluxon generation that $X(\infty)>0$. (In fact, a large value of $t$ is chosen for the estimation for the generation.) For $\kappa_{P}(t)$ we choose a symmetrical linear increase and decrease

$$
\begin{array}{ll}
\kappa_{P}(t)=0 & \text { for }|t|>t_{0}, \\
\kappa_{P}(t)=\left(\kappa_{p o} / t_{0}\right)\left(t_{0}-|t|\right) & \text { for }|t|<t_{0} .
\end{array}
$$

Figure 1 shows the results of the soliton trajectory, i.e., $u$ vs $X$ relations when $\kappa_{\mathrm{dc}}=1.8, t_{0}=2.5, \alpha=0.02$, and $\beta=0$. As shown in Fig. $1(\mathrm{a})$, when $\kappa_{p 0}=0.30$, the soliton returns to the initial position, i.e., $X(\infty)=X_{0}$, meaning that a fluxon is not generated. On the other hand, when $\kappa_{P 0}$ is increased up to 0.325 , the fluxon enters in the junction. From

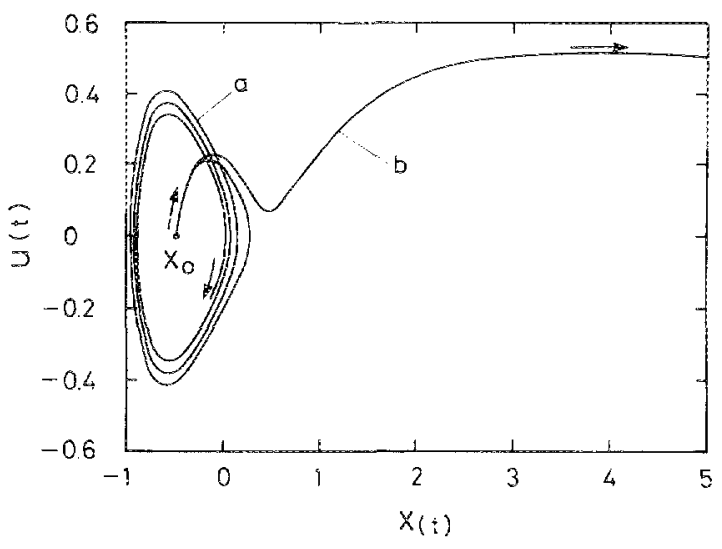

FIG. 1. $u$ vs $X$ curves, representing fluxon trajectories in the generation process. $\alpha=0.02, \beta=0, \kappa_{\mathrm{dc}}=1.8$, and $t_{0}=2.5$. (a) $\kappa_{P 0}=0.30$ and (b) $\kappa_{i \neq 0}=0.325$.

these procedures it is found that the criteria for the fluxon generation at $\kappa_{\mathrm{dc}}=1.8$ exist between $\kappa_{p 0}=0.30$ and 0.325 . The solid curves in Fig. 2 show the criteria of a fuxon generation, where $\kappa_{\mathrm{dc}}$ is plotted versus the puise height $\kappa_{p 0}$ at the threshold for various values of $t_{0}$ and for $\alpha=0.02$ and $\beta=0$.

To examine the validity of our perturbation calculation we have performed a direct numerical integration of $\mathrm{Eq}$. (1) with the initial condition, Eqs. (3) and (4). For $\kappa_{P}(t)$ we use the same form as in the perturbation calculation Eq. (16). The integration is carried out by expressing the long junction by the lumped circuit model and solving the ordinary differential equations of the model by means of the Runge-Kutta routine. ${ }^{7}$ The result of this numerical integration is shown in the dashed curves in Fig. 2.

We see that the deviation between the two results (both curves in Fig. 2) is very small for $\kappa_{\mathrm{dc}} \leqslant 2$, meaning that the perturbation treatment is adequate for such conditions. The general feature is the following. All curves start in $\left(\kappa_{P 0}, \kappa_{\mathrm{de}}\right)$ $=(0,2)$ with an aimost linear decrease.

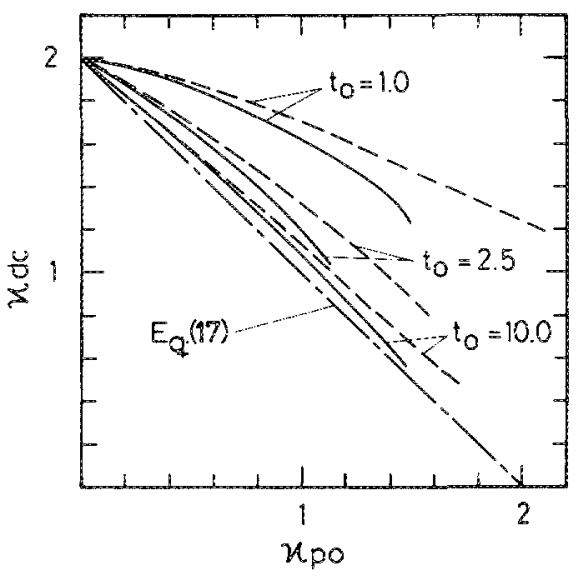

FIG. 2. Threshold curve for a fluxon generation obtained by the perturbation treatment (solid curves), and the direct numerical integration (dashed curves). Shown is the de part of the current $\kappa_{\mathrm{dc}}$ vs the pulse height $\kappa_{P O}$ for various values of the pulse width $t_{0}$, when $\alpha=0.02$ and $\beta=0$. 
For large pulse duration the curves approach the adiabatic $\left(t_{0}=\infty\right)$ curve, i.e.,

$$
\kappa_{\mathrm{dc}}+\kappa_{p \mathrm{O}}=2
$$

because the application of very slow pulse corresponds to the quasistatic increase of the dc current. The terminations observed in Fig. 2 are due to the fact that the trial function soliton for sufficiently high pulses reaches the velocity 1 .

Finally we derive the initial linear decrease of $\kappa_{\mathrm{de}}$ vs $\kappa_{P 0}$ in Fig. 2 by simple but different arguments. We have $\kappa_{\text {tc }} \approx 2$ and $\kappa_{P 0} \approx 0$.

The following criterion of the generation is imposed: if the fluxon is centered inside the junction at $t=0$ (at which the pulse has the maximum $\kappa_{P 0}$ ), then it is generated, but otherwise, it is not generated. Then, using the velocity $u_{0}$ at $t=0$, the generation condition can be expressed as

$$
\left|X_{4}\right| \geqslant u u_{0} t_{3}
$$

where $\mu$ is a constant depending on the pulse shape and height. Furthermore, the trial function is assumed precisely to follow the boundary condition for $\Phi_{x}[\mathrm{Eq}$. (2)] at $x=0$. This means that we have at $t=0$

$$
\kappa_{\mathrm{dc}}+\kappa_{\mathrm{p} 0}=2 \gamma\left(u_{0}\right)
$$

In Eq. (4) we can replace $\operatorname{sech}\left(X_{0}\right)$ by $1-X_{0}^{2} / 2$ because $\kappa_{\mathrm{dc}} \approx 2$, and in Eq. (19) $\gamma\left(u_{0}\right)$ by $1+u_{0}^{2} / 2$ because
$\kappa_{P O} \approx 0$. Using Eq. (18) and eliminating $u_{0}$ and $X_{0}$ from Eqs. (4) and (19) we obtain the following threshold curve:

$$
\kappa_{\mathrm{sc}}+\kappa_{p \mathrm{v}} \mu^{2} t_{0}^{2} /\left(1+\mu^{2} t_{0}{ }^{2}\right)=2
$$

Note that Eq. (20) is consistent with Eq. (17) as $t_{0} \rightarrow \infty$. If we put $\mu=1 / 2$, Eq. (20) explains the initial slopes of $\kappa_{\mathrm{dc}}$ vs $\kappa_{P 0}$ curves very well. $\mu=1 / 2$ corresponds to a constant acceleration (linear velocity increase). Different pulse shape will lead to different $\mu$ values.

One of us (S.S.) is grateful for the hospitality of the personnel in Physics Laboratory 1 , the Technical University of Denmark.

${ }^{1}$ See, for example, A. Barone and G. Paterno, Physics and Applications of the Josephson Effect (Wiley, New York, 1978).

${ }^{2} \mathrm{~S}$. Sakai, H. Akoh, and H. Hayakawa, Jpn. J. Appl. Phys. 23, L610 (1984); 24, L749 (1985); 24, L771 (1985).

${ }^{3}$ D. W. McLaughlin and A. C. Scott, Phys. Rev. A 18, 1652 (1978).

${ }^{\circ} O$. H. Olsen, N. F. Pedersen, M. R. Samuelsen, H. Svensmark, and D. Welner, Phys. Rev. B 33, 168 (1986).

5O. H. Olser and M. R. Samuelsen, J. Appl. Phys. 52, 6247 (1981).

The model of this letter is assumed to have an ideal current source. As can be seen in $\mathrm{Eq}$. (9), however, this theory is extendable ro models with other kinds of sources (e.g., a voltage source with a resistor).

${ }^{7}$ S. N. Erne and R. D. Parmentier, J. Appl. Phys. 51, 5025 (1980). 